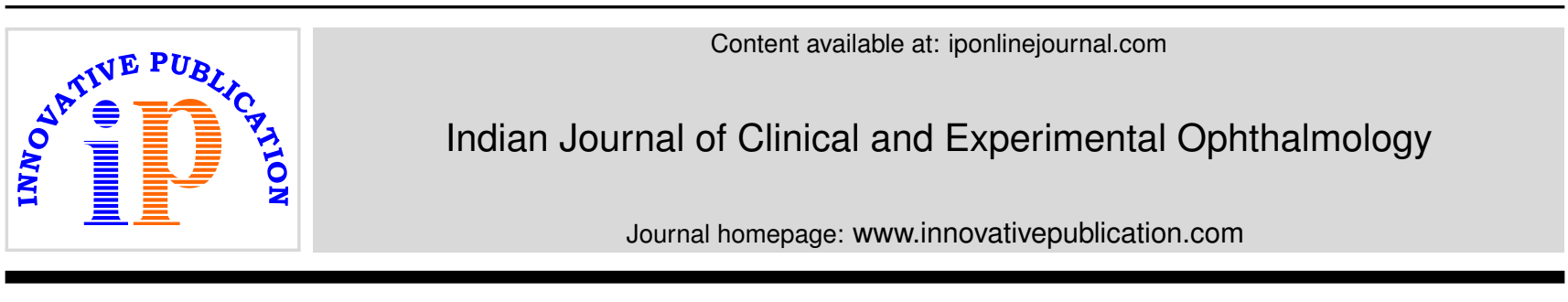

\title{
Editorial
}

\section{Ocular oncology practice during SARS-CoV-19 related lockdown}

\author{
Rajendra P Maurya ${ }^{1}$,* \\ ${ }^{1}$ Regional Institute of Ophthalmology, Institute of Medical Sciences, Banaras Hindu University, Varanasi, Uttar Pradesh, India
}

\section{A R T I C L E I N F O}

Article history:

Received 30-05-2020

Accepted 02-06-2020

Available online 16-06-2020
(C) 2020 Published by Innovative Publication. This is an open access article under the CC BY-NC license (https://creativecommons.org/licenses/by-nc/4.0/)
Beta corona virus disease is a relatively new entity caused by Severe Acute Respiratory Syndrome Coronavirus-2 (SARS-CoV-2); also known as 2019 novel corona virus disease (COVID-19) was first identified in China on December 3, 2019. ${ }^{1,2}$ In India COVID-19 was detected first time on January 30, 2020 Since, then it has spread across the globe to become one of the most serious pandemics. ${ }^{3}$ The World Health Organization (WHO) has declared COVID-19 outbreak as a public health emergency of international concern on January 30, 2020. ${ }^{4}$ By June 2 , 2020, its outbreak was wide spread internationally causing 6,28,7771 infections and 3,79,941 deaths in several counties. In India, the total number of confirmed COVID19 cases exceeded 2,07615 and 5845 deaths as of June 2, 2020.5

The human-to-human transmission is confirmed. As compared with other types of corona virus like SARS \& MERS, this virus has high risk of transmissibility and infectivity, despite of low mortality rate. ${ }^{6,7}$ The virus is mainly transmitted through direct contact of respiratory droplets or by touching a contaminated surface or objects via nose, mouth and eye. ${ }^{8}$ Transmission via ocular surface is also reported. The transmission due to aerosol contact with conjunctiva in unprotected eyes has also been reported. ${ }^{9,10}$

Elderly, immunocompromised patients and patients with pre-existing co-morbidities like diabetes, cardiovascular diseases, hypertension and cancer are at greatest risk of mortality. Along with cancer it has become the second leading cause of death worldwide. COVID-19 infection is likely to increase cancer related mortality to a great extent. Wenhua Liang et at. analyzed 2007 cases from 575 hospitals in China and found that patients with cancer had higher risk of severe COVID-19 infection than those without cancer. ${ }^{11}$ McGoogan WZ reported that COVID-19 fatality rate was about $5.6 \%$ among cancer patients as compared with $2.3 \%$ in general population. ${ }^{12}$ Cancer patient are prone to COVID-19 because of immunocompromised condition due to both malignancy and anticancer chemotherapy.

The only way to prevent virus infection/transmission is by social distancing and isolation, to control virus epidemic, due to unavailability of effective treatment and vaccine. In Indian prospective, the complete lockdown was enforced at midnight of March 24, 2020 with total travel bans and schools and universities were indefinitely closed. ${ }^{13}$ During lockdown all routine outpatient departments across the hospitals and clinics were to be shut and only emergency services continuing to function. The COVID-19 outbreak is the leading unprecedented consequence on cancer care. It may have direct or remote consequences on patients and health care providers. The Ocular oncology practice during the COVID-19 pandemic related lockdown is quite challenging. Cases of COVID-19in India are still increasing at a great pace even during $5^{\text {th }}$ lockdown, started from June $1,2020$.

Recent Chinese study shows that most of the cancer patients are at a higher risk in developing severe events in COVID-19 due to immunosuppressed status. ${ }^{7}$ Many patients of ocular malignancies are also immunocompro- 
Table 1: Levels of urgency in ocular oncology according to AAOOP recommendations

\section{Levels of Urgency}

Emergent

Urgent

\section{Semiurgent}

\section{Nonurgent}

\section{Procedures in Ocular Oncology}

1. Orbital biopsy for malignancy in a child (suspected rhabdomyosarcoma)

2. Enucleation for intractable glaucoma /globe perforation from intraocular tumor (retinoblastoma, uveal melanoma).

1. Examination under anaesthesia for newly suspected retinoblastoma.

2. Enucleation for retinoblastoma.

3. Orbital biopsy for processes causing optic neuropathy and vision loss.

4. Orbital decompression for impending visual loss (optical neuropathy or corneal perforation) secondary to orbital tumor.

1. Examination under anaesthesia for children with active retinoblastoma undergoing treatment (intravenous chemotherapy, intra-arterial chemotherapy, intravitreal chemotherapy, plaque radiotherapy, cryotherapy, transpupillary thermotherapy, laser photocoagulation) must continue on necessary schedule to control disease, typically every 3-4 weeks.

2. Examination under anaesthesia for retinoblastoma evaluation for patients with stable disease, who have received treatment within past 6 months.

3. Examination under anaesthesia for children at high risk for retinoblastoma due to family history or unknown RB gene mutation.

4. Intraocular injection of chemotherapeutic agents for high-grade neoplasia.

5. Biopsy of suspected intraocular malignancy (fine needle aspiration biopsy or other).

6. Excision /drainage of iris cyst with pain or glaucoma.

7. Plaque insertion and removal for posterior uveal Melanoma (choroidal \& cilliary body).

8. Tantalum clip insertion for posterior uveal Melanoma (Choroidal \& cilliary body).

9. Enucleation for uveal Melanoma.

Eyelid Tumors

10. Biopsy of suspected eyelid malignancies including Melanoma, sabaceous carcinoma, Merkel cell carcinoma or others.

11. Excision of suspected malignant tumor or orbital tumor affecting the better eye in a monocular patient (slow-growing I lead basal cell carcinoma should be excised on a non urgent basis).

12. Repair of island effect after tumor removal.

Conjunctival Tumors

13. Biopsy of suspected conjunctival malignancy including Melanoma and squamous cell carcinoma which could not be managed reasonably without patient topical chemotherapy of the patieent.

14. Biopsy of suspected conjunctival lymphoma (extended may be appropriate under certain circumstances).

Orbital Tumors

15. Biopsy of suspected tumor malignancy (case by case-rapidly growing tumor may need urgent biopsy, slowly growing suspected lymphoma is semiurgent).

16. Biopsy of suspected orbital lymphoma (extended delay may be appropriate under certain circumstances).

17. Exenteration (case by case rapidly growing tumor may need urgent biopsy; slowly growing one could be semiurgent).

1. Biopsy of suspected benign eyelid tumor.

2. Biopsy of suspected basal cell carcinoma, unless monocular patient.

3. Biopsy of suspected benign conjunctival tumor.

4. Biopsy of suspected benign orbital tumor.

5. Treatment of select iris Melanoma with excision or radiation therapy (some may be urgent, especially if there is a rapid growth or secondary glaucoma, at physician's discretion).

6 . Excision /drainage of cyst without pain or glaucoma. 
Table 2: Practical health vigilance recommendation adopted by modena cancer centre

Concerns

Patient care

Facility reorganisation

\section{Recommendations}

1. Provide surgical face masks and access to cleaning/disinfecting agents for all people having access to the clinic.

2. Contact patient on active anti cancer treatment the day before the appointment for screening of symptoms of cough, sore throat, fever or other flu-like symptoms. Patient with symptoms must be rescheduled.

3. Allow access to visitors only if essential for visit.

4. Prohibit visitors' access to the haemopoietic stem cell transplantation unit and the inpatient hematology ward.

5. Postpone routine follow-up visits for patient not on active cancer treatment (e.g 6-12 months survivorship visits), and conduct appointment via telemedicine.

6. Respect lifestyle restrictions

1. Limit access to the hospital through one point of entry.

2. Establish triagestations outside the hospital to screen patients and visitors for covid-19 symptoms.

3. Convert the waiting area to allow for distancing of at least $1 \mathrm{~m}$.

4. Dedicated and confined hospital area for patients with respiratory symptoms to present to the oncological / hematological admission room (eg. COVID-19 room).

1. Provide personal protective equipment (PPE)(e.g. surgical face mask) and access to cleaning /disinfecting agents for all the staff members.

Staff
2. Train clinic staff for symptom recognition, screening procedures and PPE use.

3. Reduce the number of non-medical staff in the clinic by allowing work from home for phone based activities.

4. Organize web-based multi-disciplinary team meetings.

5. Respect lifestyle restrictions. mised from cancer therapy and are at risk of contracting COVID-19. The patients of active retinoblastoma receiving intra arterial chemotherapy are at major risk due to associated neutropaenia. ${ }^{14,15}$ As the pandemic worsens, there is a need to reduce the risk of virus transmission and to protect the patients of ocular cancer and their attendants or family members accompanying a minor and healthcare provider including doctor and paramedical staff. Therefore the major strategies should be postponing of adjuvant therapies, elective surgeries, routine follow-up and cancer prevention appointment for less aggressive ocular cancers. However delaying and denying oncological interventions may lead to progressive disease, nonresectable tumor leading to poor survival particularly in potential fatal ocular malignancies such as retinoblastoma, orbital rhabdomyosarcoma and uveal Melanoma etc. ${ }^{16}$ Thus oncologist should provide only urgent or emergent care during covid-19. The cancer care cannot be postponed only on the basis of the risk of infection. Prioritization of cases must be done according to guidelines for triage for cancer surgery patients by American College of surgeons. ${ }^{17}$

A guideline for prioritization of ocular oncology cases given in Table 1. [Recommendations endorsed by Board of Directors of American Association of Ophthalmic Oncologist and Pathologists (AAOOP) and the International Society of Ocular Oncology (ISOO)] must be followed. ${ }^{18}$ According to (AAOOP) recommendations ocular oncology cases have been divided into (i) Emergent (should be performed within 24hr) (ii) Urgent (should be performed within a week), (iii) Semi urgent (should be performed within one to two months) and (iv) Non urgent (should be deferred for at least two to three months). In the COVID19 pandemic situation malignant tumors are given priority over benign tumors and highly malignant tumors leading to death or disability like retinoblastoma, uveal melanoma and rhabdomyosarcoma etc are prioritized over less aggressive malignancies like basal cell carcinoma (BCC) of eyelid and OSSN etc. ${ }^{18}$

The patients who are at low risk, benign and non urgent tumors are given the choice of telemedicine consultation or to delay their visit and treatment. The general survey with reference to Indian prospective reported that $77.5 \%$ ophthalmologist could use some form of telemedicine which could be in the form of a simple phone call or a video- call, short message service (SMS) and whatsup chat. Telemedicine helps to decide whether patient requires urgent intervention or not. ${ }^{19}$ Documentation of phone calls and telemedicine consultation in medical record is essential. Ideal ocular oncology cases for telemedicine consultation are conjunctival lymphoma, OSSN, benign conjunctival melanosis (being externally located and slow growing) and benign or low malignant eyelid tumors like eyelid BCC. ${ }^{20}$ In COOG survey (collaborative ocular oncology group survey) $100 \%$ respondents were managed 
new patients as with active disease (having proptosis /Pain) within last 3 months as emergency. ${ }^{21}$

Interventions like detailed examination of retinoblastoma under general anesthesia where intubation is necessary or surgical procedures in which possibilities of aerosolization of virus carries high risk for transmission of SARSCoV-2 requiring special precautions, including presurgical COVID-19 testing, use of full PPE kit etc.to minimize the risk of virus exposure to healthcare providers. Modena Cancer Centre adopted practical health vigilance recommendation for cancer management (Table 2) which is to be followed during ocular oncology procedures. ${ }^{22}$ Many institutions adopted COVID-19 testing of patients and their care taker /family members before admission. Sometimes second round PCR testing is also required due to high level of false negative results.

Protection of professionals is most sensitive issue during cancer management in covid-19 pandemic. Several reports are available from India where health care workers got COVID-19 infection during cancer care. On April 7, 2020 in Delhi State Cancer institute (DSCI), 18 health workers including two doctors were found COVID-19 positive. $^{23}$ On April 14, 2020 two doctors and 2 nurses were found COVID-19 infected in Asian Cancer institute, Mumbai. ${ }^{24}$ Thus preventive barriers are mandatory for all professionals involved in cancer care.

\section{Source of Funding}

None.

\section{Conflict of Interest}

None.

\section{References}

1. Lu H, Stratton CW, Tang YW. Outbreak of pneumonia of unknown etiology in Wuhan, China: The mystery and the miracle. J Med Virol. 2020;92(4):401-2.

2. Zhu N, Zhang D, Wang W. A novel corona virus from patient with pneumonia in China. N Eng J Med. 2019;382(8):727-33.

3. Honavar S, Khanna R. All eyes on Coronavirus-What do we need to know as ophthalmologists. Indian J Ophthalmol . 2020;68(4):549-53.

4. Available from: https://Www.who.int/emergencies/diseases/ novelcoronavirus 20 g.

5. 2020. Available from: https://www.worldometers.Info/coronavirus

6. Chen Y, Liu Q, Guo D. Coronaviruses: genome structure replication and pathogenesis. J Med Virol. 2020;92:418-23.

7. Li Q, Guan X, P. Early transmission dynamics in Wuhan , China, of novle coronavirus infected pneumonia. $N$ Engl J Med. 2020;382(13):1199-1207.

8. Kampf G, Todt D, Pfaender S, Steinmann E. Persistence of coronaviruses on inanimate surfaces and their inactivation with biocidal agents. J Hosp Infect . 2020;104(3):246-51.

9. Lu CW, Liu XF, Jia ZF. 2019-nCoV transmission through the ocular surface must not be e ignored. Lancet. 2020;395:39.
10. Available from: https://www.aao.org/headline/coronavırus-killschinese-whistleblower-ophthalmol

11. Liang W, Guan W, Chen R. Cancer patients in SARS-CoV-2 infection nationwide analysis in China. Lancet Oncol. 2020;21(3):335-7.

12. Wu Z, Mcgoogan JM. Characteristic of and important lesson from the coronavirus disease 2019 (covid-19) outbreak in China: Summary of a report of 72314 cases from the Chinese centre for disease control and prevention. JAMA. 2020;.

13. Available from: https://www.bbc.com/news/world-asia-india52024239.

14. Abramson DH, Daniels AB, Marr BP, Francis JH, Brodie SE, Dunkel IJ, et al. Intra-Arterial Chemotherapy (Ophthalmic Artery Chemosurgery) for Group D Retinoblastoma. PLOS ONE. 2016;11(1):e0146582.

15. Funes S, Sampor C, Villasante F, Fandiño A, Manzitti J, Sgroi M, et al. Feasibility and results of an intraarterial chemotherapy program for the conservative treatment of retinoblastoma in Argentina. Pediatr Blood Cancer. 2018;65(8):e27086.

16. Cortiula F, Pettke A, Bartoletti M, Puglisi F, Helleday T. Managing COVID-19 in the oncology clinic and avoiding the distraction effect. Ann Oncol. 2020;31(5):553-5.

17. American College of Surgeons. COVID-19 guidelines for or triage of Cancer surgery patients. Available from: https://Www.facs.org/covid19/clinicalguidance/elective-cases/cancer-surgery.

18. Skalet AH, Allen RC, Shields CL, Wilson MW, Mruthyunjaya P, Gombos DS. Considerations for the Management and Triage of Ocular Oncology Cases during the COVID-19 Pandemic. Ocul Oncol Pathol. 2020;6(3):219-22.

19. Nair AG, Gandhi RA, Natarajan S. Effect of COVID-19 related lockdown on ophthalmic practice and patient care in India: Results of a survey. 2020;68(5):725-30.

20. Stuart A. Covid-19 pandemic: ocular tumor triage and Care Eye Net; 2020. Available from: https://Www.aao.org/eyenet/article/covid-19pandemic-ocular-tumor-triage-and-care

21. Collaborative ocular oncology group survey: Management of ocular oncology patients during the COVID-19 Pandemic. Available from: https://castlebiosciences.com/wp-content/uploads/2020/ D3/COOG-Consensus-COVIDI9_2020-03-26.pdA

22. Omarini C, Maur M, Luppi G, Narni F, Luppi M, Dominici M, et al. Cancer treatment during the coronavirus disease 2019 pandemic: Do not postpone, do it! Eur J Can. 2020;133:29-32.

23. Available from: https://Www.hidustantimes.com//April2020.

24. Available from: hitps://tımesofindia.Indiatımes.com/covid-19 14 april2020.

\section{Author biography}

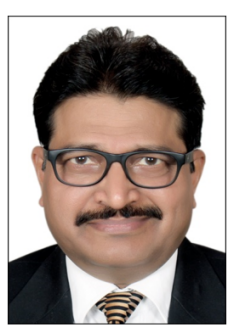

Rajendra P Maurya (MS, Ph.D) Editor in Chief IJCEO, Senior Assistant Professor \& I/c Orbit, Ocular Oncology and Oculoplasty Unit Regional Institute of Ophthalmology, Institute of Medical Sciences, Banaras Hindu University, Varanasi, (UP), India E-mail: editorijceo@gmail.com, mauryarp_bhu@yahoo.com

Cite this article: Maurya RP. Ocular oncology practice during SARS-CoV-19 related lockdown. Indian J Clin Exp Ophthalmol 2020;6(2):155-158. 